\section{An auroral flare at Jupiter}

\section{J. H. Waite Jr*, G. R. Gladstone $\dagger$, W. S. Lewis $\dagger$, R. Goldstein $\dagger$, D. J. McComas $\dagger$, P. Riley $\ddagger$, R. J. Walker $\$, P. Robertson $\dagger$, S. Desaill, J. T. Clarke ${ }^{\star}$ \&. T. Young*}

* Department of Atmospheric, Oceanic, and Space Sciences, University of Michigan, Ann Arbor, Michigan 48109, USA

$\dagger$ Southwest Research Institute, PO Drawer 28510, San Antonio, Texas 78228, USA

$\ddagger$ SAIC, 10260 Campus Point Drive, San Diego, California 92121, USA

$\$$ Institute of Geophysics and Planetary Physics, UCLA, Los Angeles, California 90095, USA

|| 1305 Addiewell Place, San Jose, California 95120, USA

Jupiter's aurora is the most powerful in the Solar System ${ }^{1}$. It is powered largely by energy extracted from planetary rotation ${ }^{2}$, although there seems also to be a contribution from the solar wind $^{3,4}$. This contrasts with Earth's aurora, which is generated through the interaction of the solar wind with the magnetosphere. The major features of Jupiter's aurora (based on far-ultraviolet ${ }^{5-7}$, near-infrared ${ }^{8,9}$ and visible-wavelength ${ }^{10}$ observations) include a main oval that generally corotates with the planet and a region of patchy, diffuse emission inside the oval on Jupiter's dusk side. Here we report the discovery of a rapidly evolving, very bright and localized emission poleward of the northern main oval, in a region connected magnetically to Jupiter's outer magnetosphere. The intensity of the emission increased by a factor of 30 within $70 \mathrm{~s}$, and then decreased on a similar timescale, all captured during a single four-minute exposure. This type of flaring emission has not previously been reported for Jupiter (similar, but smaller, transient events have been observed at Earth), and it may be related directly to changes in the solar wind.

Four imaging observations of Jupiter's northern far-ultraviolet aurora were performed with the Hubble Space Telescope Imaging Spectrograph (STIS) in time-tagged mode during a roughly twohour period on 21 September 1999 (Fig. 1). Time-tagged images obtained during the second observation, between 21:04:50 and 21:08:50 UT, reveal a dramatic, rapidly intensifying, flare-like auroral emission at jovicentric latitudes between $60^{\circ}$ and $70^{\circ}$ (Fig. 2). These latitudes lie poleward of the main oval, which generally coincides with the ionospheric footprint of magnetic field lines that map to corotating regions in the magnetic equatorial plane; the outer boundary of these regions is though to lie near $30 R_{\mathrm{J}}$ where $R_{\mathrm{J}}$ is the radius of Jupiter ${ }^{5-7}$. The event begins as a small, 'pinpoint' emission near $167^{\circ}$ System III longitude and $63^{\circ} \mathrm{N}$ (frames $1-3$ ), which rises rapidly in intensity and becomes a structure several thousand kilometres across (frames 5-12). The event reaches its maximum intensity at $\sim 70 \mathrm{~s}$ (frame 8 ), after which it starts to decrease in both intensity and size (frames 9-14 and 19-24). (STIS stopped counting temporarily during frames 15-18 owing to a full buffer. The light curve of the flare is well represented by an exponential rise (with an e-folding time of $15 \mathrm{~s}$ ) to a peak brightness of $37 \mathrm{MR}\left(1 \mathrm{MR}=10^{12}\right.$ photons $\left.\mathrm{cm}^{-2} \mathrm{~s}^{-1} 4 \pi \mathrm{sr}^{-1}\right)$ followed by an exponential decline (with a (1/e)-folding time of $63 \mathrm{~s}$ ). Other auroral emissions remain virtually unchanged in intensity and morphology during the flare event. The summed image of this event (Fig. 1b) shows that the flare occurs within a region of fainter, more diffuse emissions inside the main oval.
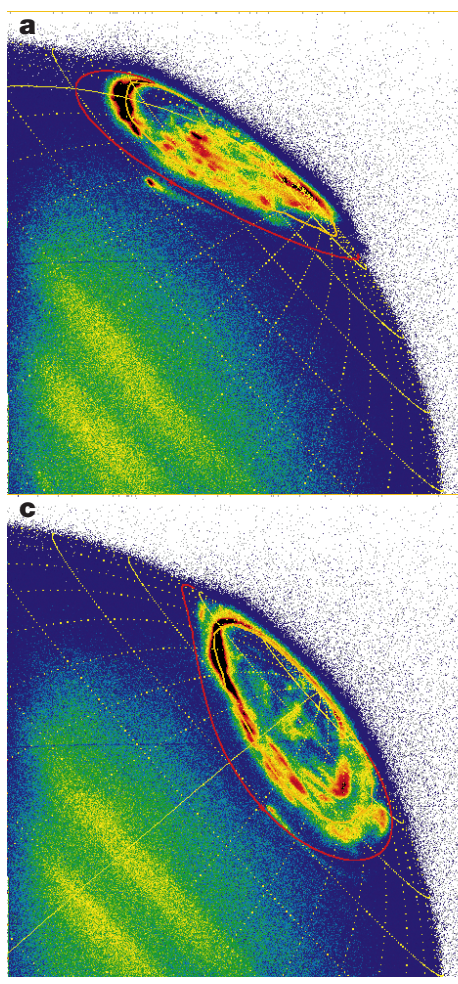

Figure 1 Hubble Space Telescope images of Jupiter's northern aurora on 21 September 1999. False-colour brightnesses are indicated in megarayleighs (MR). We have clipped the brightnesses above $1 \mathrm{MR}$ to highlight fainter emissions. The exposures were made at a, 20:27:30-20:31:30; b, 21:04:50-21:08:50; c, 22:01:54-22:06:54; and d, 22:38:5222:45:32 ut, with effective exposure times of $224.5 \mathrm{~s}, 210.5 \mathrm{~s}, 238.4 \mathrm{~s}$, and $319.5 \mathrm{~s}$, respectively. A jovicentric graticule with $10^{\circ}$ intervals is overplotted (with the $180^{\circ}$

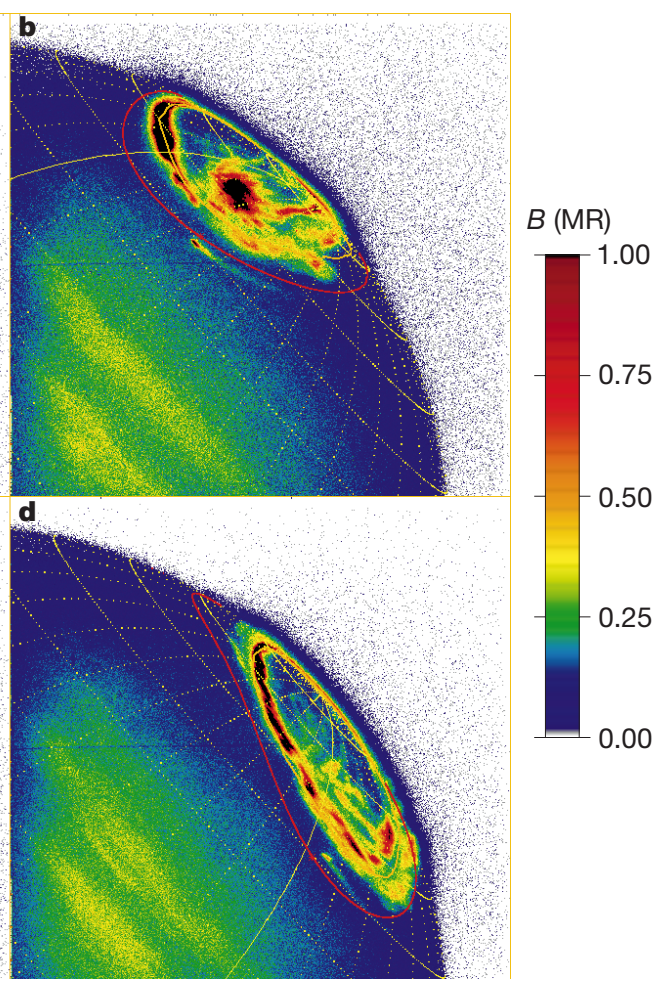

meridian in solid colour), along with $L=30$ footprint and the $L=5.9$ mapping of the lo torus. These observations were made using the F25SRF2 filter, which transmits primarily auroral emissions of the $\mathrm{H}_{2}$ Lyman bands. The sensitivity of STIS with this filter is calculated to be 0.22 counts $\mathrm{s}^{-1}$ pixel $^{-1} \mathrm{MR}^{-1}$ of $\mathrm{H}_{2}$ and $\mathrm{H}$ auroral photons emitted. 
A similar event, though shorter-lived and less intense than the flare, occurs in the first time-tagged observation as well (Fig. 1a), which was conducted half an hour earlier, from 20:27:30 to 20:31:30 UT. The most intense phase of this event lasts only about $10 \mathrm{~s}$ and reaches a maximum brightness of $17 \mathrm{MR}$. No transient brightenings are seen in the third and fourth observations (from 22:01:54 to $22: 06: 54$ UT and from $22: 38: 52$ to $22: 45: 32$ UT, respectively). However, in the summed images (Fig. 1c and d), the data show fainter but distinct localized emission features near a System III longitude of $180^{\circ}$. The relation of these features to the localized brightenings observed earlier is unknown; however, both these fainter features and the transient brightenings occur near the noon magnetic meridian, which suggests that it may be more appropriate to consider them as being organized in magnetic local time rather than System III longitude.

The four time-tagged imaging observations were performed in short segments half an hour to an hour apart and do not present a continuous picture of the evolution of polar cap auroral activity during the two-hour observation period. However, it is clear from the first time-tagged observation that polar cap activity had begun some time before 20:27:30 UT and that similar localized brightenings had occurred at the same region as the flare. In the following discussion we focus on the flare as the most dramatic of these events.

The location of the flare at latitudes poleward of the main oval indicates that it is linked by magnetic field lines to a region of the magnetosphere that lies at radial distances beyond $30 R_{\mathrm{J}}$ on Jupiter's day side. This region is not described by any of the existing jovian magnetic field models ${ }^{11,12}$. We therefore used a recently developed magnetohydrodynamic (MHD) model of the solar wind interaction with the jovian magnetosphere ${ }^{13}$ to identify the possible source region of the charged particles responsible for the flare and thus to locate the site of the magnetospheric disturbance that triggered it.

According to the model, the flare maps to a longitudinally extended region located at jovicentric distances between 40 and $60 R_{\mathrm{J}}$ in the morning sector. (Because of the corotation lag of plasma flows in the outer magnetosphere, the source region is at a somewhat earlier local time than is the flare on the planet.) It can be assumed the flare was triggered by a disturbance in this region of the magnetosphere. However, the nature and cause of such a disturbance is not known.
Unfortunately, no in situ data on conditions in the day side magnetosphere are available for the times of our observations, which were performed while the Galileo spacecraft was located in Jupiter's dusk magnetosphere following its fly-by of Callisto five days earlier, on 16 September. The Galileo magnetometer data show no unusual conditions at the time of the Hubble Space Telescope (HST) observations (K. Khurana, personal communication). Other Galileo particles and fields data are not available for this day.

Likewise, there are no near-Jupiter solar-wind data available for this time period. However, using a one-dimensional, time-dependent eulerian finite-difference fluid $\operatorname{code}^{14,15}$, we propagated the one-hour averaged solar-wind data from the Solar Wind Electron Proton Alpha Monitor (SWEPAM) ${ }^{16}$ on the Advanced Composition Explorer (ACE) spacecraft at L1-proton speed, density, and dynamic pressure - from $1 \mathrm{AU}$ to the location of Jupiter at $4.9 \mathrm{AU}$. The results (Fig. 3) show a series of sharp rises in the solar-wind dynamic pressure at the orbit of Jupiter near the time that the flare

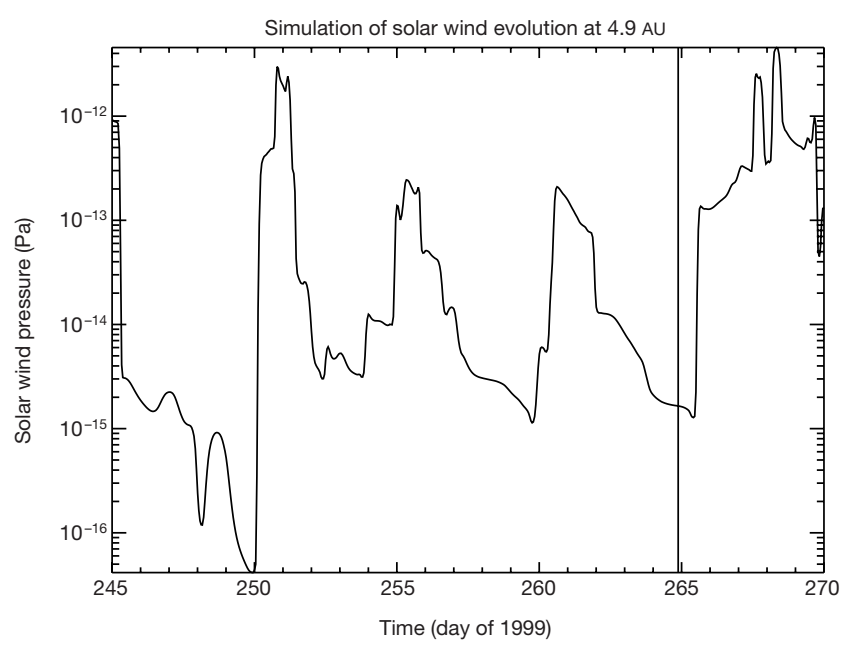

Figure 3 Modelled solar wind dynamic pressure at Jupiter. The calculated solar wind plasma pressure during days 245-270,1999, is based on spacecraft measurements at Earth's L1 point (Earth was less than $45^{\circ}$ from Jupiter as seen from the Sun at the time of the observations). The vertical line marks the flare event time at Jupiter.

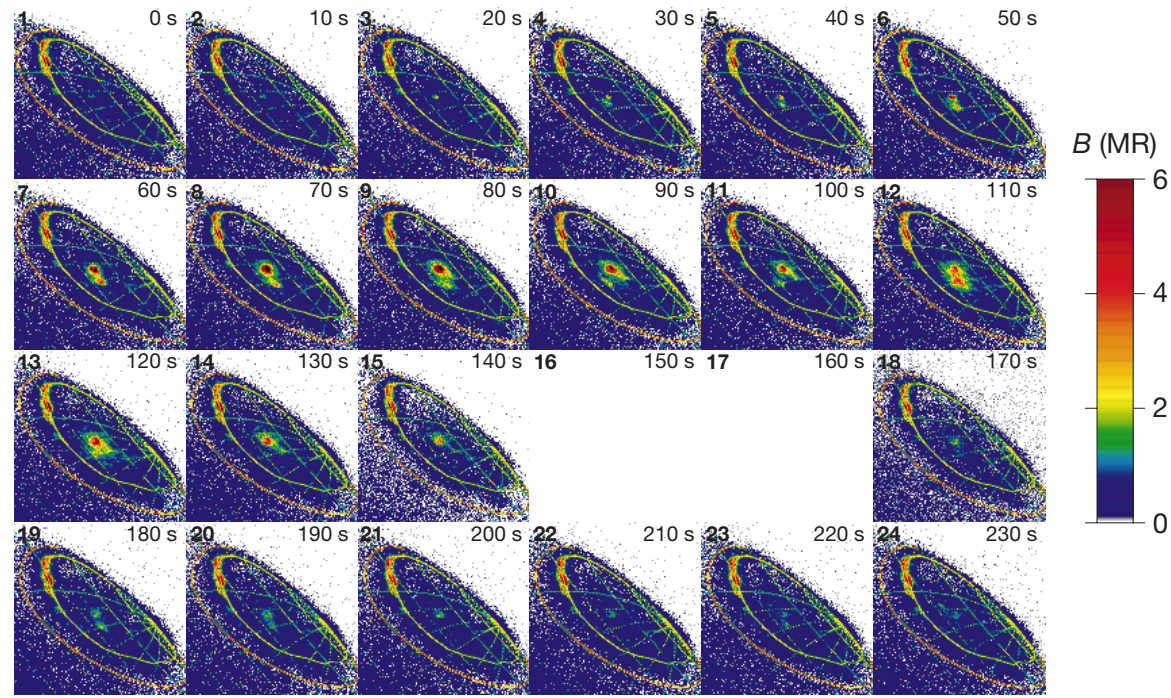

Figure 2 The rapidly evolving auroral flare observed poleward of Jupiter's auroral oval. The time series shows the evolution of the flare beginning at 21:04:50 ut on 21 September. Ten-second frames are used, and the brightness scale is as indicated (with brightnesses clipped at 6 MR). The peak brightness of the flare at the 70-s frame is $37 \mathrm{MR}$ (total $\mathrm{H}_{2}+\mathrm{H}$ emissions). An animated version of these observations can be viewed as Supplementary Information, and at http://pluto.space.swri.edu/yosemite/jupiter/flare.html. 
was observed. Jupiter's magnetosphere has been known since the Pioneer encounters to be highly compressible and to vary rapidly and dramatically in size with changes in solar wind dynamic pressure $^{17}$. Moreover, previous studies have demonstrated a correlation between changes in solar-wind pressure and changes in the intensity of auroral emissions at both infrared and radio wavelengths $s^{3,4}$. Although uncertainties in the simulation, and the fact that Jupiter and ACE were offset from each other by about $43^{\circ}$ in ecliptic longitude, prevent us from identifying an exact solar-wind feature to match the flare observation, it is not unreasonable to speculate that a sharp jump in dynamic pressure at Jupiter's day side magnetopause produced the disturbance that manifested itself in the polar-cap flare. The solar-wind conditions at this time were not unusual, which suggests that such flares, if indeed triggered by changes in solar-wind pressure, may not be uncommon. We note that responses of Earth's aurora to solar-wind dynamic pressure pulses are well known, both as rapid global brightenings associated with the passage of interplanetary shocks ${ }^{18,19}$ and as smaller-scale transient auroral events observed in the day side cusp/cleft region ${ }^{20}$. Thus similar physical processes may occasionally be at work in both the terrestrial and the jovian auroras, although the transient intensification observed at Jupiter is much more powerful than similar events observed at Earth.

A unique opportunity to test the possible role of solar-wind pressure pulses in triggering polar-cap transients was presented by the Cassini fly-by of Jupiter in December 2000. Coordinated Cassini, HST and Galileo observations were planned to provide near-simultaneous monitoring of solar-wind conditions (Cassini), auroral activity (HST), and magnetospheric conditions (Galileo). The results of these coordinated observations are expected to provide additional new examples of the puzzling new auroral feature reported here and to yield new insights into jovian auroral phenomena and the underlying magnetospheric processes.

Received 4 October 2000; accepted 1 March 2001

1. Bhardwaj, A. \& Gladstone, G. R. Auroral emissions of the giant planets. Rev. Geophys. 38, 295-353 (2000).

2. Hill, T. W., Dessler, A. J. \& Goertz, C. K. in Physics of the Jovian Magnetosphere (ed. Dessler, A. J.) 353 394 (Cambridge Univ. Press, Cambridge, 1983).

3. Desch, M. D. \& Barrow, C. H. Direct evidence for solar wind control of Jupiter's hectometerwavelength radio emission. J. Geophys. Res. 89, 6819-6823 (1984).

4. Baron, R. L., Owen, T., Connerney, J. E. P., Satoh, T. \& Harrington, J. Solar wind control of Jupiter's $\mathrm{H}_{3}^{+}$ auroras. Icarus 120, 437-442 (1996)

5. Clarke, J. T. et al. Far-ultraviolet imaging of Jupiter's aurora and the Io "footprint". Science 274, 404409 (1996).

6. Clarke, J. T. et al. Hubble Space Telescope imaging of Jupiter's UV aurora during the Galileo orbiter mission. J. Geophys. Res. 103, 20217-20236 (1998).

7. Prangé, R. et al. Detailed study of FUV Jovian auroral features with the post-COSTAR HST faint object camera. J. Geophys. Res. 103, 21095-20215 (1998).

8. Connerney, J. E. P., Baron, R., Satoh, T. \& Owen, T. Images of excited $\mathrm{H}_{3}^{+}$at the foot of the Io flux tube in Jupiter's atmosphere. Science 262, 1035-1038 (1993).

9. Satoh, T. \& Connerney, J. E. P. Jupiter's $\mathrm{H}_{3}^{+}$emissions viewed in corrected jovimagnetic coordinates. Icarus 141, 236-252 (1999).

10. Vasavada, A. R. et al. Jupiter's visible aurora and Io footprint. J. Geophys. Res. 104, 27133-27142 (1999).

11. Connerney, J. E. P., Acuña, M. H., Ness, N. F. \& Satoh, T. New models of Jupiter's magnetic field constrained by the Io flux tube footprint. J. Geophys. Res. 103, 11929-11939 (1998)

12. Khurana, K. K. Euler potential models of Jupiter's magnetospheric field. J. Geophys. Res. 102, 11295 11306 (1997)

13. Ogino, T., Walker, R. J. \& Kivelson, M. G. A global magnetohydrodynamic simulation of the jovian magnetosphere. J. Geophys. Res. 103, 225-235 (1998).

14. Stone, J. M. \& Norman, M. L. ZEUS-2D: A radiation magnetohydrodynamics code for astrophysical flows in two space dimensions. I. The hydrodynamic algorithms and tests. Astrophys. J. Suppl. Ser. 80, 753-790 (1992).

15. Gosling, J. T. \& Riley, P. The acceleration of slow coronal mass ejections in the high-speed solar wind. Geophys. Res. Lett. 23, 2867-2870 (1996).

16. McComas, D. J. et al. Solar wind electron proton alpha monitor (SWEPAM) for the Advanced Composition Explorer. Space Sci. Rev. 86, 563-612 (1998).

17. Intriligator, D. S. \& Wolfe, J. H. in Jupiter: Studies of the Interior, Atmosphere, Magnetosphere, and Satellites (ed. Gehrels, T.) 848-869 (Univ. Arizona Press, Tucson, 1976).

18. Craven, J. D., Frank, L. A., Russell, C. T., Smith, E. J. \& Lepping, R. P. in Solar Wind-Magnetosphere Coupling (eds Kamide, Y. \& Slavin, J. A.) 367-380 (Terra Scientific, Tokyo, 1986).

19. Zhou, X. \& Tsurutani, B. T. Rapid intensification and propagation of the dayside aurora: Large scale interplanetary pressure pulses (fast shocks). Geophys. Res. Lett. 26, 1097-1100 (1999).
20. Sandholt, P. E. et al. Cusp/cleft auroral activity in relation to solar wind dynamic pressure, interplanetary magnetic field $\mathrm{B}_{\mathrm{z}}$ and $\mathrm{B}_{\mathrm{y}}$. J. Geophys. Res. 99, 17323-17342 (1994).

Supplementary information is available on Nature's World-Wide Web site (http://www.nature.com).

\section{Acknowledgements}

We gratefully acknowledge discussions with B. T. Tsurutani and J. L. Burch and the support of the Space Telescope Science Institute.

Correspondence and requests for materials should be addressed to J.H.W (e-mail: hunterw@umich.edu).

\section{Quantum computing in molecular magnets}

\section{Michael N. Leuenberger \& Daniel Loss}

Department of Physics and Astronomy, University of Basel, Klingelbergstrasse 82, 4056 Basel, Switzerland

Shor and Grover demonstrated that a quantum computer can outperform any classical computer in factoring numbers ${ }^{1}$ and in searching a database ${ }^{2}$ by exploiting the parallelism of quantum mechanics. Whereas Shor's algorithm requires both superposition and entanglement of a many-particle system ${ }^{3}$, the superposition of single-particle quantum states is sufficient for Grover's algorithm ${ }^{4}$. Recently, the latter has been successfully implemented ${ }^{5}$ using Rydberg atoms. Here we propose an implementation of Grover's algorithm that uses molecular magnets ${ }^{6-10}$, which are solid-state systems with a large spin; their spin eigenstates make them natural candidates for single-particle systems. We show theoretically that molecular magnets can be used to build dense and efficient memory devices based on the Grover algorithm. In particular, one single crystal can serve as a storage unit of a dynamic random access memory device. Fast electron spin resonance pulses can be used to decode and read out stored numbers of up to $10^{5}$, with access times as short as $10^{-10}$ seconds. We show that our proposal should be feasible using the molecular magnets $\mathrm{Fe}_{8}$ and $\mathrm{Mn}_{12}$.

Suppose we want to find a phone number in a phone book consisting of $N=2^{n-1}$ entries. Usually it takes $N / 2$ queries on average to be successful. Even if the $N$ entries were encoded in binary, a classical computer would need approximately $\log _{2} N$ queries to find the desired phone number ${ }^{2}$. But the computational parallelism provided by the superposition and interference of quantum states enables the Grover algorithm to reduce the search to one single query ${ }^{2}$. Here we will show that this query can be implemented in terms of a unitary transformation applied to the single spin of a molecular magnet. Such molecular magnets, forming identical and largely independent units, are embedded in a single crystal so that the ensemble nature of such a crystal provides a natural amplification of the magnetic moment of a single spin. However, for the Grover algorithm to succeed, it is necessary to find ways to generate arbitrary superpositions of spin eigenstates. For spins larger than $1 / 2$ this turns out to be a highly non-trivial task as spin excitations induced by magnetic dipole transitions in conventional electron spin resonance (ESR) can occur only in discrete steps of one $\hbar$ (Planck's constant divided by $2 \pi$ ), that is, single steps by two or more $\hbar$ values are excluded by selection rules. To circumvent such physical limitations we propose an unusual scenario which, in principle, allows the controlled generation of arbitrary spin superpositions through the use of multifrequency coherent magnetic 